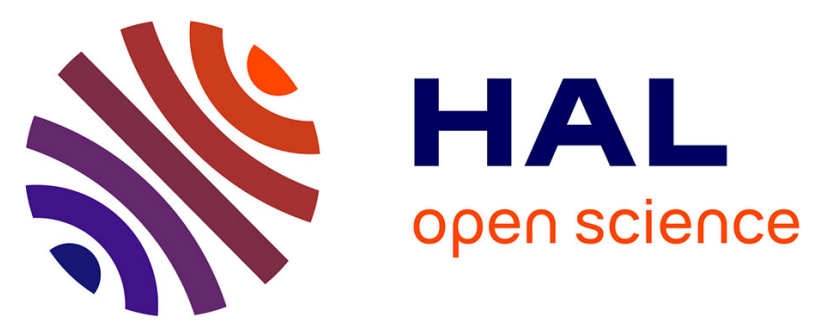

\title{
Sociocognitive determinants of self-reported compliance with standard precautions: Development and preliminary testing of a questionnaire with French health care workers
}

Estelle Michinov, Sylvie Buffet-Bataillon, Cindy Chudy, Aymery Constant, Veronique Merle, Pascal Astagneau

\section{To cite this version:}

Estelle Michinov, Sylvie Buffet-Bataillon, Cindy Chudy, Aymery Constant, Veronique Merle, et al.. Sociocognitive determinants of self-reported compliance with standard precautions: Development and preliminary testing of a questionnaire with French health care workers. American Journal of Infection Control, 2016, 44 (1), pp.14-19. 10.1016/j.ajic.2015.07.041 . hal-01214013

HAL Id: hal-01214013

https://hal-univ-rennes1.archives-ouvertes.fr/hal-01214013

Submitted on 14 Jan 2016

HAL is a multi-disciplinary open access archive for the deposit and dissemination of scientific research documents, whether they are published or not. The documents may come from teaching and research institutions in France or abroad, or from public or private research centers.
L'archive ouverte pluridisciplinaire HAL, est destinée au dépôt et à la diffusion de documents scientifiques de niveau recherche, publiés ou non, émanant des établissements d'enseignement et de recherche français ou étrangers, des laboratoires publics ou privés. 


\title{
Sociocognitive determinants of self-reported compliance with standard precautions: Development and preliminary testing of a questionnaire with French health care workers.
}

\author{
Estelle Michinov, $\mathrm{PhD}^{\mathrm{a}}$, , Sylvie Buffet-Bataillon, $\mathrm{PD}, \mathrm{PhD}^{\mathrm{b}}$, Cindy Chudy ${ }^{\mathrm{a}}$, Aymery \\ Constant, $\mathrm{PhD}^{\mathrm{c}}$, Véronique Merle, $\mathrm{MD}, \mathrm{PhD}^{\mathrm{d}}$, Pascal Astagneau, $\mathrm{MD}, \mathrm{PhD}^{\mathrm{e}}$, f a \\ Department of Psychology, University of Rennes 2, Centre de Recherches en Psychologie \\ Cognition et Communication (CRPCC, E.A. 1285), Rennes, France \\ ${ }^{\mathrm{b}}$ Hospital Hygiene Unit, Rennes University Hospital, Rennes, France \\ ${ }^{c}$ Department of Social and Behavioral Sciences, EHESP School of Public Health, \\ Sorbonne Paris Cité, France \\ ${ }^{\mathrm{d}}$ Department of Epidemiology and Public Health, Rouen University Hospital, Rouen, \\ France \\ ${ }^{\mathrm{e}}$ Centre de Coordination pour la Lutte contre les Infections associées aux soins (C-CLIN), \\ Paris, France \\ ${ }^{\mathrm{f}}$ EHESP School of Public Health, Sorbonne Paris Cité, France
}

\section{Highlights}

\begin{abstract}
To our knowledge, this is the first work using a sociocognitive theory to assess determinants of health care workers' self-reported compliance to standard precautions.

The sociocognitive determinants are based on the theory of planned behavior.

A preliminary questionnaire was validated with good psychometric properties.

The questionnaire can be used to adapt infection prevention strategies to medical and nursing staff.
\end{abstract}

\section{Background}

Inconsistent compliance of health care workers with standard precautions has already been documented. The objective of this study was to develop a questionnaire to investigate the sociocognitive determinants of compliance with standard precautions based on the theory of planned behavior.

\section{Methods}

To construct the Standard Precautions Questionnaire (SPQ), items were selected using a systematic review of literature and semistructured interviews with 54 health care workers. Thirty-five items were selected for a draft questionnaire. These questionnaires were sent to 649 health care workers in 3 medical specialties (pediatrics, geriatrics, and intensive care) in a French University hospital. A total of 331 valid questionnaires were analyzed. 


\section{Results}

Factor analysis yielded a final 7-factor solution with an explained variance of $66.51 \%$, with 24 items. The 7 dimensions were the following: attitude toward standard precautions, social influence facilitating organization, exemplary behavior of colleagues, organizational constraints, individual constraints, and intention to perform standard precautions. Some differences were observed between medical specialties on attitude toward standard precautions, social influence, and individual constraints.

\section{Conclusion}

The SPQ met the conditions of reliability and validity in accordance with psychometric demands and could be used to evaluate attitudes and intention to perform standard precautions among medical and nursing staff.

\section{Key Words}

- Infection control;

- Standard precautions;

- Theory of planned behavior;

- Scale development

Effective methods for changing the infection control practices of health care workers are needed to reduce health care-associated infections and improve patient safety. To reduce infection rates, the Centers for Disease Control and Prevention revised its guidelines for infection control practice in 1996, replacing universal precautions with standard precautions. Standard precautions are infection prevention practices that apply to all patient care, regardless of the suspected or confirmed infection status of the patient, in any setting where health care is delivered. ${ }^{1 \text { and } 2}$ Their aim is to reduce the risk of cross-transmission of microorganisms to ensure the safety of patients and caregivers by reducing contact with blood and body substances through the implementation of safe work practices and protective barriers. They include the following: (1) hand hygiene; (2) using disposable gloves; (3) using appropriate personal protective equipment during procedures and patient-care activities; (4) appropriate procedures for contaminated material; (5) transport and management of waste; and (6) cleaning and disinfection of contaminated surfaces. ${ }^{1,2,3}$ and 4

The compliance of health care workers with hygiene practices has been extensively investigated in the last 10 years, with compliance ranging from $<30 \%$ in hand hygiene to $>90 \%$ in appropriate disposal of sharps. ${ }^{5,6 \text { and } 7}$ In recent observational audits in France, adherence to hand hygiene practice after removal of gloves varies from $17.5 \%$ during nursing procedures to $93 \%$ when bathing dependent patients, with a compliance of $63.6 \%$ with standard precautions. ${ }^{8}$

Although health care workers recognize standard precautions as an important health careassociated infection prevention measure, putting them into routine practice could be jeopardized by work conditions (lack of time), infrastructure (lack of equipment, poor facility design), type of patient contact, inadequate training and knowledge, social environment (superiors, colleagues, patients), and safety climate. ${ }^{9}$ Studies of health care workers' compliance with standard precautions have found that knowledge and social factors (eg, 
safety climate, organizational campaigns) have a positive influence on attitudes and behaviors regarding infection control and prevention. ${ }^{10,11 \text { and } 12}$

The interdependence of individual factors, environmental constraints, and organizational climate could play a major role in behavioral intentions regarding hygiene precautions. ${ }^{13}$ and 14 To understand attitudes to and strategies of compliance, a large number of studies have investigated the determinants of compliance with hygiene practice. Some of these studies have used the theory of planned behavior ${ }^{15}$ and 16 as a theoretical framework to develop a model of adherence to hygiene recommendations. ${ }^{17}$ and 18 This theory of planned behavior appears to offer an appropriate way of predicting behavior. ${ }^{19}$ This theory postulates that behavioral intention (an individual's readiness to perform a behavior) is determined by 3 intermediate variables: attitude toward the behavior, subjective norms, and perceived behavioral control. Attitude concerns the degree to which an individual has a favorable or unfavorable evaluation of the behavior or perception that this behavior may or may not be beneficial to them. Subjective norms are related to perceived social pressure, the perceived behavior of others, and beliefs about what people think about the behavior. Perceived behavioral control is related to the perceived ease or difficulty of performing the behavior. These intermediate variables are predicted by the strength of the person's beliefs about the outcomes of the behavior, normative beliefs (perception of the expectations of peers or other social groups), and control beliefs (ability of the person to overcome obstacles or to enhance resources to facilitate the behavior). The theory of planned behavior provides a systematic framework that can be used to identify the factors associated with adherence to hygiene practice recommendations.

A number of researchers have developed questionnaires measuring the sociocognitive determinants of compliance with hand hygiene guidelines, based on the theory of planned

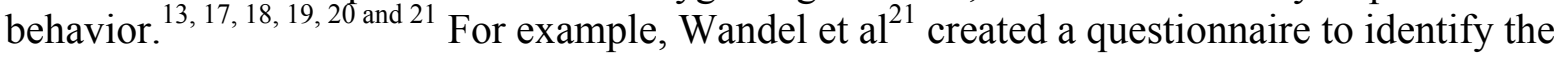
determinants of compliance with hand hygiene procedures in intensive care units. The results showed that the predictors of poor adherence were low control beliefs and negative attitudes related to lack of time and high workload. McLaws et $\mathrm{al}^{20}$ showed that adherence to hand hygiene behavior depends on peer pressure, including the exemplary behavior of senior physicians. Pittet et $\mathrm{al}^{13}$ demonstrated that hand hygiene compliance could also vary by medical specialty and type of patient contact. Finally, predictors of intent to apply hygiene recommendations may vary across studies and care settings.

A critical analysis of the literature reveals that all existing questionnaires measure attitude and intention to perform hygiene behavior. Nevertheless, to reduce infection rates and the risk of cross-contamination, it is important to understand the factors that facilitate and hinder compliance with all aspects of standard precautions. This article reports the development and preliminary validation of a questionnaire to measure the sociocognitive determinants of compliance with standard precautions among French health care workers.

\section{Methods}

We followed the 4 steps generally used for the development and preliminary validation of a questionnaire $^{22}$ : (1) specify construct domain (literature review and selection of questionnaires); (2) generate sample of items (in-depth interviews and test face validity using experts); (3) collect data (quantitative study among a representative sample); and (4) purify measure (analyze internal reliability and construct validity). 


\section{Specify domain of construct}

The construction of the questionnaire involved 2 steps: a systematic literature review, and a qualitative study with a sample of French health care workers (in-depth interviews). For a systematic literature review, we followed the Preferred Reporting Items for Systematic Reviews and Meta-Analyses guidelines. ${ }^{23} \mathrm{We}$ conducted a systematic search for original articles in the MEDLINE, PsycINFO, PsycARTICLES, and Psychology \& Behavioral Sciences Collection databases. Only English language articles and peer-reviewed journals were selected. The major key words used to search from 1995-2013 were standard precautions and theory of planned behavior and scale development or handwashing and theory of planned behavior and scale development. The last search was run on December 16, 2013. We selected items from the Integrated quality Criteria for systematic Reviews Of Multiple Study designs ${ }^{24}$ tool to assess the quality of the included studies: clear aims and justification, reliable questionnaire measure, questionnaire with adequate and rigorous data analysis, absence of selective outcome, and limitation addressed. The literature search was performed by 2 authors working independently of each other. The validated instruments retrieved by the literature search were mostly related to sociocognitive determinants of hand hygiene. ${ }^{13,17,18,19,20,21,25 \text { and } 26}$ No validated questionnaire measuring the sociocognitive determinants of the full spectrum of standard precautions for all health care workers was found. The few existing questionnaires related to standard precautions measured attitudes among nurses or nursing students (and not all members of medical staff) and did not evaluate the sociocognitive determinants of the full spectrum of standard precautions. ${ }^{26}$ To construct the sociocognitive determinants of the standard precautions questionnaire, we had to generate specific items.

\section{Generate sample of items}

In the second step, a psychology research assistant conducted interviews with health care workers $(N=54)$ in 3 medical specialties: pediatrics $(n=17)$, geriatrics $(n=19)$, and intensive care $(\mathrm{n}=18)$. These specialties were selected because patients in these units are particularly vulnerable to health care-associated infections. The severity of their illness, the presence of a high number of invasive devices, and frequent contact with health care workers are common risk factors. ${ }^{17}$ Semistructured interviews with different caregivers (registered nurses, assistant nurses, physicians and charge nurses) were carried out around 3 themes related to the sociocognitive determinants of behavioral intention: (1) attitudes, (2) subjective norms, and (3) behavioral control. For attitudes, participants were asked if they knew the standard precautions recommendations, what they thought about their application in hospital, and what they thought about the importance of applying standard precautions. For subjective norms, participants were asked if their colleagues encouraged (or did not encourage) them to follow the standard precautions protocol. For behavioral control, participants were asked which factors facilitated and which hindered the application of standard precautions. All interviews were conducted and transcribed verbatim by a psychology research assistant. Analysis followed the approach of content analysis. ${ }^{27}$ The content analysis data are available as unpublished data and can be accessed by contacting the authors.

The literature review of existing instruments and the content analysis of the interviews allowed us to create an initial draft questionnaire with 35 items. The first dimension of the questionnaire was related to attitude and contained 6 items (eg, if I follow the standard precaution protocol, I will protect my patients from infection; standard precautions are always effective in reducing health care infections); the second dimension was related to social 
influence and subjective norms and contained 9 items (eg, most of my colleagues think that it is important to use standard precautions; I will be reprimanded by the physicians if I do not use standard precautions); the third dimension was related to facilitators of compliance with standard precautions, which had 7 items (eg, factors that facilitate the application of standard precautions: availability of equipment in all patient care areas; to be trained in using standard precautions; when my colleagues show exemplary behavior regarding standard precautions); the fourth dimension measured barriers to using standard precautions (eg, factors that make it difficult to use standard precautions: lack of time; increased workload; complexity of the standard precaution protocol); and the final dimension was behavioral intention, which was measured by 4 items (eg, it is my intention to comply with the hospital's standard precaution protocol: even if the patient is difficult; even if I am pressed for time). The respondents' profile was also described through the following variables: age, sex, medical specialty, professional category, and length of professional experience. The Standard Precaution Questionnaire (SPQ) is available on request to the authors.

This first draft was pretested with 14 nurses (10 nurse assistants and 4 registered nurses). This pretest led to some modifications of the questionnaire (5-point Likert scale for all items; reformulation of some items; elimination of redundant items). Finally, to make all the items clear and unambiguous (test of face validity), 3 experienced infection control practitioners and 2 academic psychologists were asked to review each item. Ambiguous and complex terms were either removed or rephrased.

\section{Data collection}

The 35-item SPQ was tested on a representative sample of health care workers. Sample size was based on the need to perform a factor analysis, which required a minimum of 300 health care workers and 5-10 participants per item. ${ }^{28}$ and 29 The charge nurses in 3 medical specialties (pediatrics, geriatrics, and intensive care) in a French university hospital handed out the questionnaire to 649 health care workers during staff meetings in June 2014. Along with the paper-and-pencil questionnaire, participants received an accompanying letter that explained the purpose of the survey: a study on standard precautions (ie, you have day-to-day contact with patients, and, as such, we would appreciate your personal opinion of standard precautions). They were instructed to place the completed questionnaires in an urn for confidentiality of responses. The mean time for completing the 24-item instrument was 6 minutes.

Two weeks after the distribution of the questionnaires, a psychology research assistant made follow-up telephone calls to the charge nurse or head physician, or met the staff. The study protocol was approved by the local ethics committee, and informed consent was obtained from all participants.

\section{Data analysis}

Statistics were performed with SPSS 20.0 software (IBM, Armonk, NY). The questionnaire was evaluated for reliability and construct validity. The internal consistency was assessed by Cronbach $\alpha$ coefficient. This is the most widely used indicator of reliability in the literature. ${ }^{22,}$ 23, 24, 25, 26, 27 and 28 Construct validity was assessed by exploratory factor analysis, which was performed using oblimin rotation, allowing the factors to be correlated. ${ }^{28}$ Before performing the exploratory factor analysis, the adequacy of data for factor analysis was assessed using the Kaiser-Meyer-Olkin measure of sampling adequacy and the Bartlett test of sphericity, which 
tests the suitability of the correlation matrix for factor analysis. ${ }^{28}$ and 29 For the factor analysis, the Kaiser-Meyer-Olkin value should be $>0.50$, the cutoff level for the communality value should be 0.45 , and the criterion for factor extraction should be eigenvalue $>1{ }^{28}$ Pearson product-moment correlation coefficient was also used to evaluate concurrent validity of the questionnaire. The scores of each dimension of the SPQ in the different medical specialties (geriatric, pediatric, and intensive care) were also compared using 1-way analysis of variance. Bonferroni post hoc tests were used to compare means. For all tests, a significance level of .05 was used, and the exact level is given in all results.

\section{Results}

\section{Demographic characteristics of the sample}

Of the original 649 questionnaires, 331 were completed and collected. The overall response rate was therefore $51 \%$, with $46.55 \%$ in pediatrics (135/290), $52.90 \%$ in intensive care $(82 / 155)$, and $55.88 \%$ in geriatrics $(114 / 204)$. The overwhelming majority of the participants were women $(87.9 \%)$. The participants' mean age \pm SD was $35.16 \pm 9.27$ years, with a median of 34 years. Average time working in the department \pm SD was $5.84 \pm 6.49$ years, with a median of 4 years. Participants were mainly nurses (registered nurses: $49.5 \%$; assistant nurses: $30.2 \%$; charge nurses: $9.6 \%$ ) and physicians and medical students $(9.6 \%)$; missing data were $0.9 \%$.

\section{Factor analysis and structure of the questionnaire}

Before the exploratory analysis, the Kaiser-Meyer-Olkin measure and Bartlett test of sphericity were used to measure the sampling adequacy. The results showed that the KaiserMeyer-Olkin value was 0.75 , and the significance of the Bartlett sphericity was $<.05$ $\left(\chi^{2}=3904.67\right)$. These results indicated that the data were fit for exploratory factor analysis. Factor analysis yielded a 10 -factor solution with an explained variance of $65.23 \%$, with eigenvalues $>1$. To define the composition of the 10 factors, items that did not load strongly or cleanly on a single factor were eliminated. Seven items (items 1, 13, 14, 15, 16, 20, and 27) were deleted at this stage because they did not load strongly $(<0.45)$ on a single factor but on several factors at the same time or because they saturated on no factor. Moreover, the analysis of internal consistency of factors derived from this first component analysis revealed that 4 items should be deleted because of poor Cronbach $\alpha$ coefficients (items 5 and 6 for the attitude factor; items 11 and 12 for the social influence factor).

The remaining 24 items were entered into a second exploratory factor analysis. The results yielded a Kaiser-Meyer-Olkin value of 0.73 , indicating that the sample met the criteria for factor analysis. Factor analysis yielded a final 7-factor solution with an explained variance of $66.51 \%$, with eigenvalues $>1$. All items loaded on the expected factors, and the variance was 0.50 for all items (Table 1$)$. 
Table 1 Factor loadings and factor structure of the Standard Precautions Questionnaire

Factors

\section{Items}

To prevent infection

Protect patients from infection

Protect me from infection Influence of colleagues

Influence of charge nurse

Influence of infection control nurses

Influence of physicians

Facilities available in

health care setting

Training in use of SP

Reminders about SP

Exemplary behavior of

medical manager

Exemplary behaviour of

colleagues

Occurrence of

unanticipated events

Lack of time

\begin{tabular}{|c|c|c|c|c|c|c|}
\hline 1 & 2 & 3 & 4 & 5 & 6 & 7 \\
\hline \multirow[t]{11}{*}{$\begin{array}{c}\text { Exemplary } \\
\text { behavior }\end{array}$} & $\begin{array}{l}\text { Organizational } \\
\text { constraints }\end{array}$ & Intention & $\begin{array}{c}\text { Social } \\
\text { influence }\end{array}$ & Attitude & $\begin{array}{l}\text { Facilitating } \\
\text { organization }\end{array}$ & $\begin{array}{l}\text { Individual } \\
\text { constraints }\end{array}$ \\
\hline & & & & 0.73 & & \\
\hline & & & & 0.85 & & \\
\hline & & & & 0.78 & & \\
\hline & & & 0.61 & & & \\
\hline & & & 0.84 & & & \\
\hline & & & 0.82 & & & \\
\hline & & & 0.76 & & & \\
\hline & & & & & 0.75 & \\
\hline & & & & & 0.82 & \\
\hline & & & & & 0.68 & \\
\hline \multicolumn{7}{|l|}{0.88} \\
\hline \multicolumn{7}{|l|}{0.88} \\
\hline & 0.69 & & & & & \\
\hline & 0.90 & & & & & \\
\hline
\end{tabular}


Factors

\begin{tabular}{|c|c|c|c|c|c|c|c|}
\hline Items & 1 & 2 & 3 & 4 & 5 & 6 & 7 \\
\hline & $\begin{array}{l}\text { Exemplary } \\
\text { behavior }\end{array}$ & $\begin{array}{l}\text { Organizational } \\
\text { constraints }\end{array}$ & Intention & $\begin{array}{c}\text { Social } \\
\text { influence }\end{array}$ & Attitude & $\begin{array}{c}\text { Facilitating } \\
\text { organization }\end{array}$ & $\begin{array}{l}\text { Individual } \\
\text { constraints }\end{array}$ \\
\hline Heavy workload & & 0.88 & & & & & \\
\hline $\begin{array}{l}\text { Complexity of the SP } \\
\text { protocol }\end{array}$ & & 0.55 & & & & & \\
\hline $\begin{array}{l}\text { Lack of knowledge about } \\
\text { SP }\end{array}$ & & & & & & & -0.70 \\
\hline Care team routine & & & & & & & -0.69 \\
\hline Personal beliefs about SP & & & & & & & -0.80 \\
\hline $\begin{array}{l}\text { Problems related to use of } \\
\text { equipment }\end{array}$ & & & & & & & -0.67 \\
\hline $\begin{array}{l}\text { Intention even if the patien } \\
\text { is difficult }\end{array}$ & & & 0.80 & & & & \\
\hline $\begin{array}{l}\text { Intention even if lack of } \\
\text { time }\end{array}$ & & & 0.89 & & & & \\
\hline $\begin{array}{l}\text { Intention even if hands are } \\
\text { damaged }\end{array}$ & & & 0.78 & & & & \\
\hline $\begin{array}{l}\text { Intention even during an } \\
\text { emergency situation }\end{array}$ & & & 0.74 & & & & \\
\hline Eigenvalue & 4.58 & 3.66 & 2.04 & 1.74 & 1.56 & 1.27 & 1.13 \\
\hline$\%$ of variance explained & 19.09 & 15.23 & 8.48 & 7.23 & 6.48 & 5.29 & 4.70 \\
\hline Cronbach $\alpha$ & 0.88 & 0.77 & 0.81 & 0.75 & 0.71 & 0.80 & 0.71 \\
\hline
\end{tabular}


Factor 1 was interpreted as reflecting exemplary behavior. This was one of the strongest factors, with the highest percentage of explained variance $(19.09 \%)$ in the SPQ. Item loading on this factor included the following 2 items: the senior physician has exemplary behavior regarding adherence to standard precautions; and my colleagues have exemplary behavior regarding adherence to standard precautions.

Factor 2 was interpreted as reflecting organizational constraints and was the other strongest factor, explaining $15.23 \%$ of variance. It included the following 4 items: the occurrence of unanticipated events that adversely affect my work, lack of time, increased workload, and the complexity of the standard precautions protocol.

Factor 3 was interpreted as reflecting intention to perform standard precautions and included the following 4 items: even if the patient is difficult, even if I am pressed for time, even if my hands are damaged or painful, and during an emergency situation.

Factor 4 was interpreted as reflecting social influence and included the following 4 items: most of my colleagues think it is important to adhere to standard precautions, I will be reprimanded by the charge nurse if I do not adhere to standard precautions, I will be reprimanded by infection control link nurses if I do not adhere to standard precautions, and I will be reprimanded by the physicians if I do not adhere to standard precautions.

Factor 5 was interpreted as reflecting attitudes toward standard precautions and included the following 3 items: standard precautions are effective in reducing health care infection; if I follow the protocol of standard precautions, I will protect my patients from infection; and following the standard precautions protocol will protect me from infection.

Factor 6 was interpreted as reflecting facilitating organization and contained the following 3 items: having equipment available in the health care setting, to be trained in using standard precautions, and to receive reminders about standard precautions.

Finally, factor 7 was interpreted as reflecting individual constraints and included the following 4 items: lack of knowledge about standard precautions, care team routine, personal beliefs about standard precautions, and problems related to use of equipment.

\section{Reliability}

The total questionnaire was found to have good internal consistency, with an $\alpha$ coefficient of 0.78. Alpha coefficients for the 7 subscales were also satisfactory and ranged from $0.71-0.88$ (Table 1).

\section{Validity}

Correlations between dimensions of the questionnaire are shown in Table 2 . The results demonstrate that the questionnaire clustered in a coherent way. Dimensions related to positive determinants of using standard precautions tended to be positively correlated. Dimensions related to negative determinants of using standard precautions tended to be negatively correlated. The correlations between dimensions were moderate. These results indicate that the questionnaire had good construct validity. 
Table 2.

Pearson correlation coefficients between dimensions of the Standard Precautions Questionnaire $(\mathrm{n}=331)$
Dimension
(1) (2) (3) (4)
(5)
(6) (7)

(1) Attitude

1

(2) Social influence

$0.17^{\dagger} 1$

(3) Facilitating organization

$0.17^{\dagger} 0.30^{\dagger} 1$

(4) Exemplary behaviour

$0.13^{*} 0.33^{\dagger} 0.48^{\dagger} 1$

(5) Organizational constraints $-0.000 .00 \quad 0.07-0.001$

(6) Individual constraints

$\begin{array}{lllll}0.08 & 0.09 & 0.25^{\dagger} 0.21^{\dagger} 0.49^{\dagger} \quad 1\end{array}$

(7) Intention

$0.040 .15^{\dagger} 0.19^{\dagger} 0.19^{\dagger}-0.19^{\dagger}-0.011$

$*$

$P<.05$.

$\dagger$

$P<.01$.

\section{Discriminating ability}

Table 3 shows scores by medical specialty, indicating that medical specialty had a significant effect on scores for attitudes toward standard precautions $\left(\mathrm{F}_{2,330}=3.80, P=.023\right)$, social influence $\left(\mathrm{F}_{2,330}=8.63, P=.0001\right)$, and individual constraints $\left(\mathrm{F}_{2,330}=3.39, P=.035\right)$. More specifically, health care workers in intensive care units had less positive attitudes toward standard precautions, social pressure by colleagues, and individual constraints than those working in pediatric units. The differences were not significant between workers in pediatric and geriatric units or between those in intensive care and geriatric units, except for social influence. For other dimensions of the questionnaire (exemplary behavior, organizational constraints, intention to perform standard precautions, and facilitating organization), there were no significant differences between medical specialties.

Table 3.

Mean scores \pm SD for each dimension of the SPQ in 3 medical specialties

\begin{tabular}{llrlrrrr}
\multicolumn{1}{c}{ Specialties } & \multicolumn{1}{c}{ EB } & OC & \multicolumn{1}{c}{ ISP } & \multicolumn{1}{c}{ SI } & \multicolumn{1}{c}{ ASP } & FO & IC \\
Intensive & $4.16 \pm$ & $3.44 \pm$ & $4.16 \pm$ & $3.68 \pm$ & $3.28 \pm$ & $4.68 \pm$ & $3.31 \pm$ \\
care & 0.76 & 0.83 & 0.56 & $0.89^{*}$ & $0.53^{*}$ & 0.55 & $0.78^{*}$ \\
& $4.37 \pm$ & $3.51 \pm$ & $4.29 \pm$ & $3.98 \pm$ & $3.46 \pm$ & $4.54 \pm$ & $3.57 \pm$ \\
Pediatrics & 0.80 & 0.83 & 0.57 & $0.72^{\dagger}$ & $0.47^{\dagger}$ & 0.63 & $0.82^{\dagger}$ \\
& $4.21 \pm$ & $3.41 \pm$ & $4.17 \pm$ & $4.15 \pm$ & $3.39 \pm$ & $4.46 \pm$ & $3.34 \pm$ \\
Geriatrics & 0.81 & 0.94 & 0.62 & $0.76^{\dagger}$ & $0.45^{* \dagger}$ & 0.62 & $0.88^{* \dagger}$
\end{tabular}


NOTE. A 1-way analysis of variance was performed to compare the mean scores of different medical specialties.

$A S P$, attitude toward standard precautions; $E B$, exemplary behavior; $F O$, facilitating organization; IC, individual constraints; ISP, intention to perform standard precautions; OC, organizational constraints; SI, social influence.

$*$

Means in the same column that do not have the same subscript differ at the .05 significance level.

$\dagger$

Means in the same column that do not have the same subscript differ at the .05 significance level.

\section{Discussion}

In this study, we developed and tested a preliminary French questionnaire to measure the sociocognitive determinants of compliance with standard precautions. This had never previously been performed to our knowledge. ${ }^{25}$ All existing questionnaires based on the theory of planned behavior are limited to hand hygiene recommendations. However, to reduce infection rates related to risks of cross-contamination, a better understanding of all behavior related to standard precautions recommendations is crucial.

The final 24-item SPQ provides promising qualities for consistency with the theory of planned behavior ${ }^{15}$ and 16 showing the classic sociocognitive dimensions related to behavioral intention: attitudes toward standard precautions, subjective norms ( 2 factors: social influence and exemplary behavior of colleagues), and perceived behavioral control (individualorganizational facilitators or constraints). All dimensions are represented by at least 3 items (except for the exemplary behavior factor, which contains 2 items) and show good internal consistency. The Cronbach $\alpha$ coefficients of all dimensions were good. Among the 7 dimensions found, 4 factors (social influence, exemplary behavior, facilitating organization, and organizational constraints) were significantly correlated to intention to comply with standard precautions recommendations. Some other factors were not related to intention to perform standard precautions, such as attitudes and individual constraints (ie, lack of knowledge, care routine, personal beliefs). In some cases, individual constraints related to knowledge and internal motivational factors were not predictive of hygiene behaviors. ${ }^{17}$ and 21 These results suggest that adherence to hygiene recommendations could be influenced more by social and environmental factors than by internal motivating factors. ${ }^{18}$ and 20

The differences observed in our survey among health care staff in 3 medical specialties, and particularly the less favorable attitude toward standard precautions and perceived behavioral control observed in intensive care units than in geriatric or pediatric units, confirm findings of previous studies. ${ }^{13 \text { and } 19}$ For example, Pittet et al ${ }^{13}$ found that social pressure to comply with hand hygiene practices was greater in geriatric and pediatric departments than in intensive care units. Godin et $\mathrm{al}^{19}$ concluded that "...behaviour adoption could be modulated by several aspects of the context, such as patients' acceptability or preference for a given treatment, 
characteristics of the health problems, new versus usual patients, patients with multiple symptoms, antecedents or counter indications for a given type of medication, etc." Further studies are warranted to examine differences in the determinants of compliance with standard precautions in other medical specialties and in different contexts of realization.

Although the present study provides a reliable and valid SPQ, it has a number of limitations. First, as with all surveys, there is the possibility of social desirability responses by health care workers, in spite of voluntary participation and guaranteed anonymity. Second, the standard precautions were not explained at the beginning of the questionnaire. Each health care worker may have had in mind a different set of behaviors. Standard precautions may be interpreted differently by different health care workers. This might limit the power of the findings and should be addressed in future studies. Third, the participants were not completely representative of the overall population of French health care workers because they were selected from only 1 hospital. There is no reason to believe that the sociocognitive determinants of using standard precautions would be significantly different in other hospitals in France. However, we will further test this questionnaire's validity and measurement invariance in other populations using confirmatory factor analysis. This may also help to establish how different study populations understand adherence to standard precautions recommendations. ${ }^{30}$ Finally, our study focused uniquely on the reliability (internal consistency), content validity (face validity), and construct validity of the SPQ (exploratory structure). Further research is needed to explore other psychometric properties of the SPQ, such as its test-retest reliability, construct validity (with confirmatory factorial analysis), predictive validity (correlation with actual hygiene behavior), responsiveness, and crosscultural validity.

\section{Conclusion}

This study provides a short questionnaire of attitudes and intention to use standard precautions. To our knowledge, it is the first time that a scale has been developed to measure the sociocognitive determinants of standard precautions practice among French health care workers. The SPQ satisfies the conditions of reliability and construct validity in accordance with psychometric demands. A sensitive and comprehensive questionnaire with good psychometric properties could be used in infection control workshops or in-service training sessions for nursing staff and students. ${ }^{26}$ Therefore, assessing attitudes and intention to perform standard precautions is important to identify facilitators and barriers. The ultimate goal is to reduce the rate of cross-infection, benefitting patients, health care workers, and organizations.

\section{Acknowledgments}

We thank the managers who contributed to organizing the study in their units (S. Kayal, C. Michelet, Y. Le Tulzo, P. Seguin, D. Somme, and P. Pladys) and all the caregivers and medical staff who took part in this study. We also thank D. Lepelletier and T. Lavigne for critical feedback in the project. 


\section{References}

1. Siegel JD, Rhinehart E, Jackson M, Chiarello L. Guideline for isolation precautions: preventing transmission of infectious agents in health care settings.

Am J Infect Control 2007;35(Suppl):S65-164.

2. World Health Organization. Standard precautions in health care: aide mémoire. Available from: http://www.who.int/csr/resources/publications/EPR_AM2_E7. pdf. Accessed March 9, 2015.

3. Curran ET. Standard precautions: what is meant and what is not. J Hosp Infect 2015;90:10-1.

4. Livshiz-Riven I, Nativ R, Borer A, Kanat-Maymon Y, Anson O. Nursing students' intentions to comply with standard precautions: an exploratory prospective cohort study. Am J Infect Control 2014;42:744-9.

5. Lam SC. Universal to standard precautions in disease prevention: preliminary development of compliance scale for clinical nursing. Int J Nurs Stud 2011;48: 1533-9.

6. Erasmus V, Daha TJ, Brug H, Richardus JH, Behrendt MD, Vos MC, et al. Systematic review of studies on compliance with hand hygiene guidelines in hospital care. Infect Control Hosp Epidemiol 2010;31:283-94.

7. Gammon J, Morgan-Samuel H, Gould D. A review of the evidence for suboptimal compliance of healthcare practitioners to standard/universal infection control precautions. J Clin Nurs 2008;17:157-67.

8. Laprugne-Garcia E, Giard M, Caillat-Vallet E, Russel I, Verjat-Trannoy D, Ertzscheid MA, et al. Evaluation de l'application des précautions standard dans les établissements de santé français. Hygiènes 2014;22:107-16.

9. Michie S, Johnston M, Abraham C, Lawton R, Parker D, Walker A. Making psychological theory useful for implementing evidence based practice: a consensus approach. Qual Saf Health Care 2005;14:26-33.

10. Kermode M, Jolley D, Langkham B, Thomas MS, Holmes W, Gifford SM. Compliance with universal/standard precautions among health care workers in rural north India. Am J Infect Control 2005;33:27-33.

11. Sax H, Uçkay I, Richet H, Allegranzi B, Pittet D. Determinants of good adherence to hand hygiene among healthcare workers who have extensive exposure to hand hygiene campaigns. Infect Control Hosp Epidemiol 2007;28:1267-74. 12. Stringer B, Haines AT, Goldsmith CH, Berguer R, Blythe J. Is use of the hands free technique during surgery, a safe work practice, associated with safety climate? Am J Infect Control 2009;37:766-72.

13. Pittet D, Simon A, Hugonnet S, Pessoa-Silva CL, Sauvan V, Perneger TV. Hand Hygiene among physicians: performance, beliefs, and perceptions. Ann Intern Med 2004;141:1-8.

14. Pessoa-Silva CL, Posfay-Barbe K, Pfister R, Touveneau S, Perneger TV, Pittet D. Attitudes and perceptions toward hand hygiene among healthcare workers caring for critically ill neonates. Infect Control Hosp Epidemiol 2005;26: 305-11.

15. Ajzen I. The theory of planned behavior. Organ Behav Hum Decis Process 1991; 50:179-211.

16. Ajzen I, Manstead AS. Changing health-related behaviors: an approach based on the theory of planned behaviour. In: Van den Bos K, Hewstone M, de Wit J, Schut H, Stroebe M, editors. The scope of social psychology: theory and applications. New York [NY]: Psychology Press; 2007. p. 43-63. 
17. O'Boyle CA, Henly SJ, Larson E. Understanding adherence to hand hygiene recommendations: the theory of planned behavior. Infect Control Hosp Epidemiol 2001;29:352-60.

18. Whitby M, McLaws ML, Ross MW. Why healthcare workers don't wash their hands: a behavioural explanation. Infect Control Hosp Epidemiol 2006;27:

484-92.

19. Godin G, Bélanger-Gravel A, Eccles M, Grimshaw J. Healthcare professionals' intention and behaviours: a systematic review of studies based on social cognitive theories. Implement Sci 2008;3:36.

20. McLaws ML, Maharlouei N, Yousefi F, Askarian M. Predicting hand hygiene among Iranian health care workers using the theory of planned behavior. Am J Infect Control 2012;40:336-9.

21. Wandel D, Maes L, Labeau S, Vereecken C, Blot S. Behavioral determinants of hand hygiene compliance in intensive care units. Am J Crit Care 2010;19:230-9.

22. DeVellis RF. Scale development theory and applications. 3rd ed. Los Angeles [CA]: Sage Publications; 2012.

23. Liberati A, Altman DG, Tetzlaff J, Mulrow C, Gøtzsche PC, Ioannidis JP, et al. The PRISMA statement for reporting systematic reviews and meta-analyses of studies that evaluate health care interventions: explanation and elaboration. PLoS Med 2009;6:e1000100.

24. Edwards R, Charani E, Sevdalis N, Alexandrou B, Sibley E, Mullett D, et al. Optimisation of infection prevention and control in acute health care by use of behaviour change: a systematic review. Lancet Infect Dis 2012;12:318-29. 25. Zomer TP, Erasmus V, van Empelen P, Looman C, van Beeck EF, Tjon-A-Tsien A, et al. Sociocognitive determinants of observed and self-reported compliance to hand hygiene guidelines in child day care centers. Am J Infect Control 2013;41: 862.

26. de Carvalho Nagliate P, Nogueira PC, de Godoy S, Mendes IA. Measures of knowledge about standard precautions: a literature review in nursing. Nurse Educ Pract 2013;13:244-9.

27. Miles M, Huberman MA. Qualitative data analysis: an expanded sourcebook. Beverley Hills [CA]: Sage Publications; 1994.

28. Tabachnick BG, Fidell LS. Using multivariate statistics. 6th ed. Upper Saddle River [NJ]: Pearson Allyn \& Bacon; 2012.

29. Hairs JF, Anderson RE, Tatham RL, Black WC. Multivariate data analysis with readings. Upper Saddle River [NJ]: Prentice Hall; 1995.

30. Pereira FM, Lam SC, Chan JH, Malaguti-Toffano SE, Gir E. Difference in compliance with Standard Precautions by nursing staff in Brazil versus Hong Kong. Am J Infect Control 2015;43:769-72. 